\title{
Long Range Interactions in Cosmology: Implications for Neutrinos
}

\section{Ivan Esteban $^{a, b, *}$ and Jordi Salvado ${ }^{c}$}

${ }^{a}$ Center for Cosmology and AstroParticle Physics (CCAPP), Ohio State University, Columbus, Ohio 43210, USA

${ }^{b}$ Department of Physics, Ohio State University, Columbus, Ohio 43210, USA

${ }^{c}$ Departament de Física Quàntica i Astrofísica and Institut de Ciències del Cosmos, Universitat de Barcelona,

Diagonal 647, E-08028 Barcelona, Spain

E-mail: esteban.6@osu.edu, jsalvado@icc.ub.edu

Sizable neutrino self-interactions could exist and affect our understanding of large neutrino density environments. But if they are mediated by a light boson, i.e., have a long range, they might be extremely challenging to probe. In this work, we scrutinize how cosmology is well-suited to study them. In particular, we demonstrate that the energy density and equation of state of interacting cosmological neutrinos would diverge from the commonly assumed ideal gas form. Our results show that the current cosmological neutrino mass bound is fully avoided in the presence of a long range interaction, opening the possibility for a laboratory neutrino mass detection in the near future. We also demonstrate an interesting complementarity between neutrino laboratory experiments and the future EUCLID survey.

*** The European Physical Society Conference on High Energy Physics (EPS-HEP2021), ***

*** 26-30 July $2021 * * *$

*** Online conference, jointly organized by Universität Hamburg and the research center DESY ***

${ }^{*}$ Speaker 


\section{Introduction}

The quest for exploring new fundamental interactions has traditionally focused on high-energy probes such as particle colliders. The main hypothesis underlying these searches is that new physics has evaded detection because it only acts at short distances or, in particle physics terms, is mediated by heavy states. However, new physics could be mediated by light particles (i.e., it could have a long range) and remain undiscovered simply because it couples too weakly to matter.

In this case, small couplings can be overcome by setups with large amounts of particles over which effects accumulate coherently. An archetypal example is gravity: despite being exceptionally weak, it was the first fundamental force discovered as it adds up over all particles in macroscopic objects. Cosmology is particularly well suited to explore this sort of many-particle effects, as in the early Universe particle number densities were extraordinarily high. Furthermore, cosmological evolution is dominated by gravity, and it is thus susceptible to be modified by any stronger interaction.

Although neutrinos are ubiquitous throughout the Universe, where many large-density environments could be affected by neutrino self-interactions, these interactions remain poorly constrained [1]. Furthermore, so far the literature has mostly ignored possible long-range effects of neutrino self-interactions [2-5] (even after the lately renewed interest due to their possible relationship to the Hubble tension [6-8] or the short baseline neutrino anomalies $[9,10])$. In this work, we systematically explore the long-range effects, finding out that they strongly affect the information about neutrino properties we extract from precise cosmological observations.

\section{Formalism and equation of state}

For simplicity, we will study scalar-mediated long range interactions among neutrinos. The action of the system is given by

$$
S=\int \sqrt{-\mathcal{G}} \mathrm{d}^{4} x\left(-\frac{1}{2} D_{\mu} \phi D^{\mu} \phi-\frac{1}{2} M_{\phi}^{2} \phi^{2}+i \bar{\psi} \not D \psi-m_{0} \bar{\psi} \psi-g \hat{\phi} \bar{\psi} \psi\right),
$$

where $\phi$ and $\psi$ are the scalar and neutrino fields respectively, $\mathcal{G}$ is the determinant of the metric and $D_{\mu}$ its associated covariant derivative, $M_{\phi}$ and $m_{0}$ are the scalar and neutrino masses respectively, $g$ is the interaction coupling, and we have used the metric signature $(-,+,+,+)$.

The equations of motion stemming from Eq. (1) give the neutrino dispersion relation [11]

$$
P_{\mu} P^{\mu}=-\tilde{m}^{2}=-\left(m_{0}+g \phi\right)^{2},
$$

i.e., when neutrinos source a classical scalar field $\phi$, they acquire an effective mass $\tilde{m} \neq m_{0}$. This scalar field, for a homogeneous and isotropic neutrino background with a momentum distribution $f_{0}(\vec{p})$, is given by

$$
M_{\phi}^{2} \phi=-g \int \mathrm{d}^{3} \vec{p} \frac{m_{0}+g \phi}{\sqrt{|\vec{p}|^{2}+\left(m_{0}+g \phi\right)^{2}}} f_{0}(\vec{p}) .
$$

If we assume that the neutrino distribution takes a Fermi-Dirac form

$$
f_{0}(\vec{p})=\frac{\mathfrak{g}}{(2 \pi)^{3}} \frac{1}{e^{|\vec{p}| / T(a)}+1},
$$


with $\mathfrak{g}$ the amount of internal degrees of freedom (including particles, antiparticles and any internal quantum number) and $T$ the neutrino temperature; we can self-consistently solve Eqs. (2) and (3) and obtain the effective neutrino mass $\tilde{m}$ as well as the equation of state parameter

$$
w \equiv \frac{P}{\rho}=\frac{-\frac{1}{2} M_{\phi}^{2} \phi_{0}^{2}+\int \mathrm{d}^{3} \vec{p} \frac{|\vec{p}|}{3 \sqrt{|\vec{p}|^{2}+\tilde{m}^{2}}} f_{0}(|\vec{p}|)}{\frac{1}{2} M_{\phi}^{2} \phi_{0}^{2}+\int \mathrm{d}^{3} \vec{p} \sqrt{|\vec{p}|^{2}+\tilde{m}^{2}} f_{0}(|\vec{p}|)} .
$$

The latter is particularly important in cosmology, as in an expanding Universe $\frac{1}{\rho} \frac{\mathrm{d} \rho}{\mathrm{d} t}=-3 H(1+w)$ with $H$ the Hubble parameter; i.e., it parametrizes the rate at which energy density is diluted.

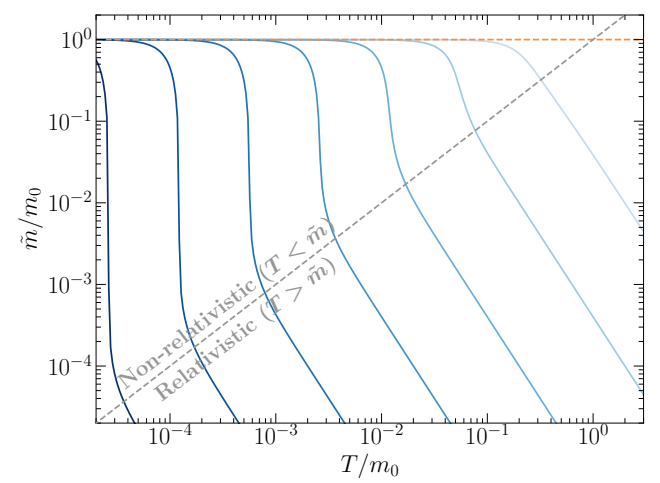

(a) Effective neutrino mass divided by $m_{0}$. The gray line separates the non-relativistic and relativistic regimes.

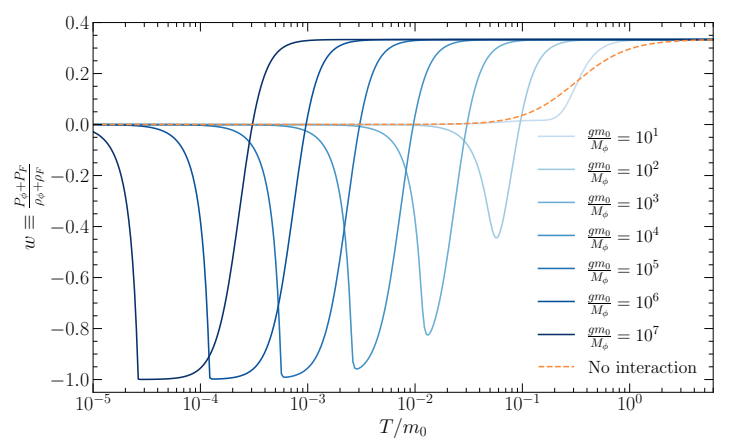

(b) Equation of state of the system.

Figure 1: Equation of state and effective neutrino mass as a function of $T / m_{0}$ for different interaction strengths [solid blue shades]. The dashed orange line shows the result without long range interactions. $T$ is the neutrino temperature, $m_{0}$ its vacuum mass, and $\frac{g m_{0}}{M_{\phi}}$ parametrizes the interaction strength. The neutrino distribution function is given by Eq. (4) with $\mathfrak{g}=6$ degrees of freedom.

Figure 1 shows that neutrino long-range interactions dramatically change their cosmological behavior. Figure 1a illustrates that the sourced scalar field reduces the effective neutrino mass, keeping them relativistic even though $T \ll m_{0}$. In Fig. $1 \mathrm{~b}$ we notice that keeping neutrinos ultrarelativistic makes them behave as radiation $(w=1 / 3)$ even for $T \ll m_{0}$. The sourced scalar field, though, also contributes to the total energy density and pressure of the system, so that $w$ can take negative values. Finally, as the system cools down the interparticle distance becomes larger than the interaction range $\sim M_{\phi}^{-1}$, and all interaction effects switch off.

\section{Cosmological data analysis}

Equations (2) and (3) modify the behavior of cosmological neutrinos. Using also the modified neutrino perturbation equations, we have carried out an analysis of present cosmological data under the presence of neutrino long-range interactions (see Ref. [11] for details). The resulting allowed regions are in Fig. 2. As we see, no neutrino mass bound can be obtained from Planck CMB data if the interaction is strong enough to delay the relativistic to non-relativistic equation of state transition (see Fig. 1a): for $g / M_{\phi} \gtrsim 10^{2} \mathrm{eV}^{-1}$, the neutrino system still behaves as radiation at recombination. For small couplings $g / M_{\phi} \lesssim 10 \mathrm{eV}^{-1}$, we essentially recover the standard cosmology and neutrino 
mass bound. Baryon acoustic oscillations (BAO) exclude a large amount of interaction strengths for relatively large values of the neutrino mass, as these would modify late-time cosmology (c.f. Ref. [11]).

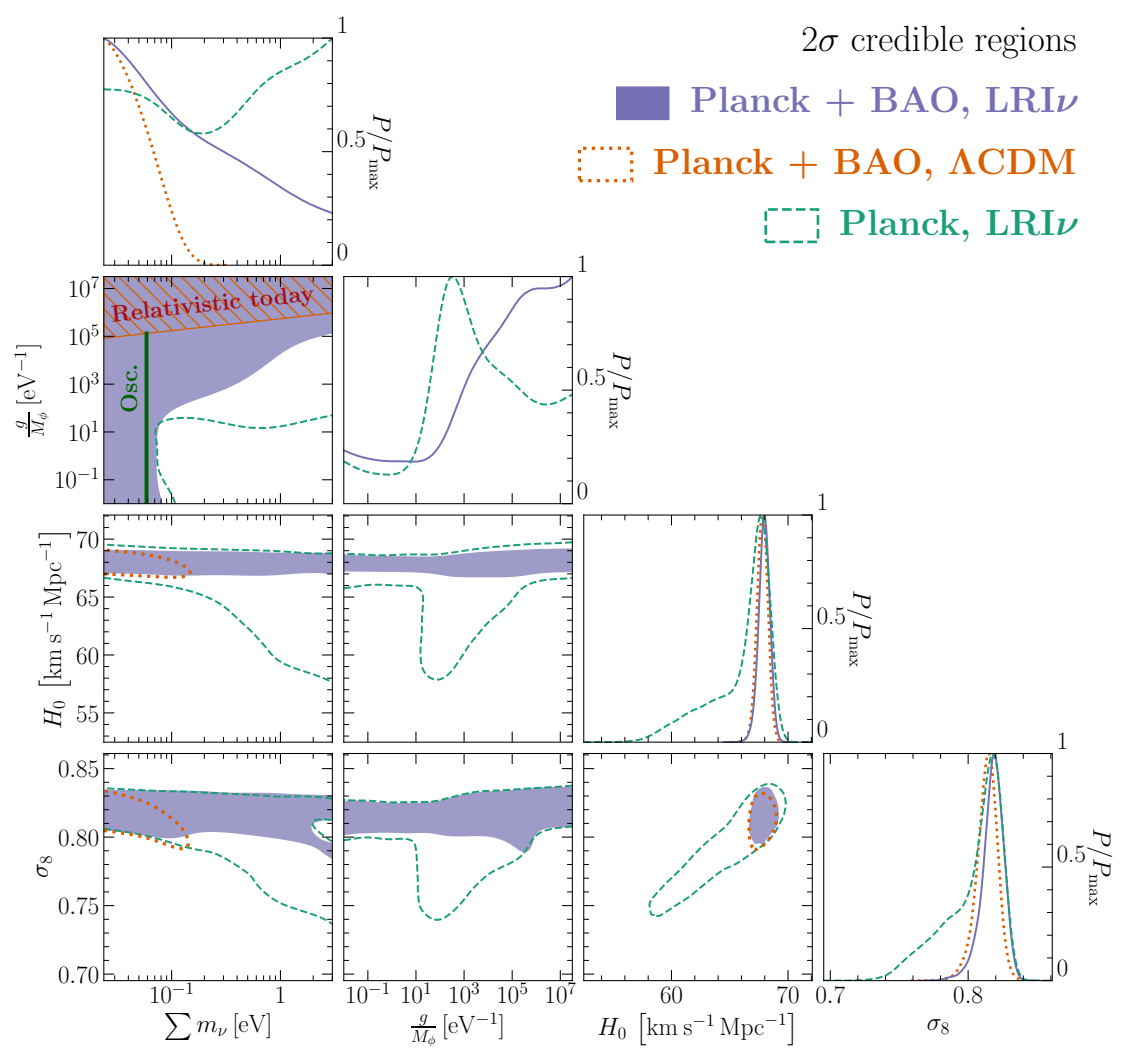

Figure 2: Planck 2018+BAO constraints [12-15] on long range interacting neutrinos, LRI $v$, [solid purple], on $\Lambda \mathrm{CDM}$ with non-interacting massive neutrinos [dotted orange], and Planck 2018 constraints [12] on long range interacting neutrinos [dashed green]. In the hatched region, cosmic neutrinos are relativistic today. The solid dark green line is the minimum value of $\sum m_{v}$ allowed by neutrino oscillation data [16].

Nevertheless, there is still no cosmological neutrino mass bound. In other words, the KATRIN laboratory experiment, that aims to constraint $\sum m_{v} \lesssim 0.6 \mathrm{eV}$ [17], could in the near future detect a non-zero neutrino mass compatible with cosmology if neutrinos have interaction strengths $g / M_{\phi} \sim 10^{3}-10^{6} \mathrm{eV}^{-1}$. In the future, though, cosmological constraints will also improve: surveys such as EUCLID [18] should have $\sim 2-3 \sigma$ sensitivity to smallest neutrino mass allowed by oscillations. To quantitatively explore the potential of EUCLID, we have carried out a forecast generating EUCLID mock data for two values of the sum of neutrino masses: $\sum m_{v}^{\text {true }}=0$, a priori excluded by oscillations; and $\sum m_{v}^{\text {true }}=0.08 \mathrm{eV}$, a value compatible with present data that should be well-measurable by EUCLID.

Figure 3 shows the future constraints on neutrino long-range interactions. As we see, EUCLID data can be consistent with massless neutrinos without violating neutrino oscillation constraints for interaction strengths $g / M_{\phi} \sim 10^{2}-10^{5} \mathrm{eV}^{-1}$. Alternatively, if EUCLID observes a neutrino mass signal, it will exclude neutrino long range interaction strengths $g / M_{\phi} \gtrsim 10^{4} \mathrm{eV}^{-1}$. Moreover, as mentioned above, a neutrino mass detection at KATRIN of $\sum m_{v} \gtrsim 0.6 \mathrm{eV}$ could point to long 


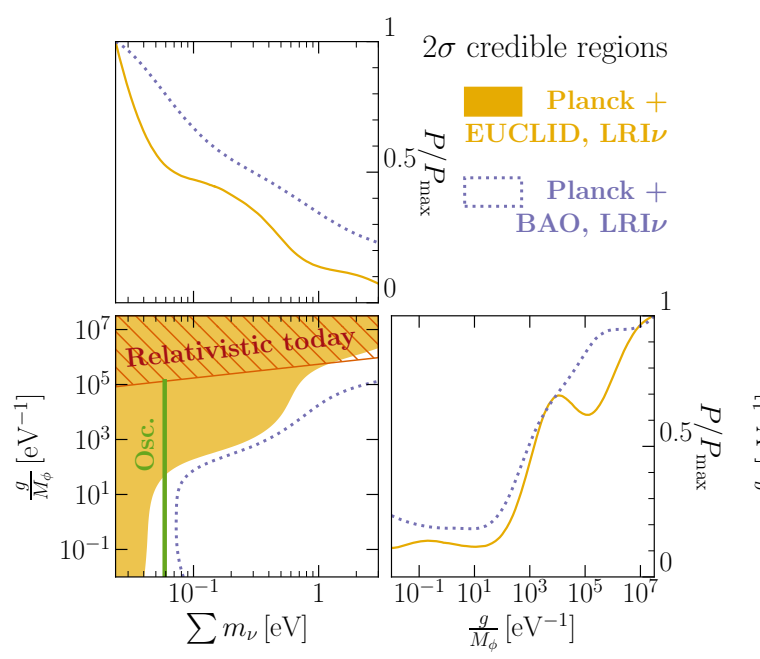

(a) $\sum m_{v}^{\text {true }}=0$

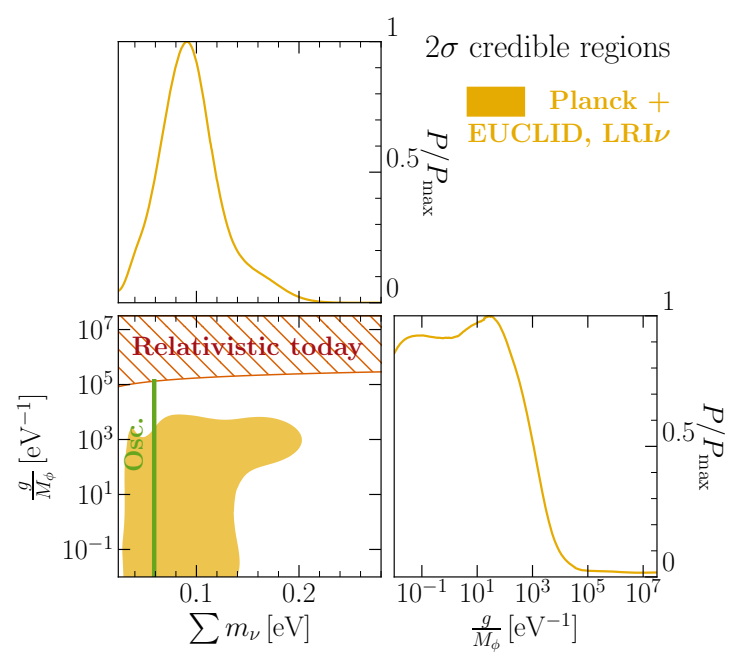

(b) $\sum m_{v}^{\text {true }}=0.08 \mathrm{eV}$

Figure 3: Future constraints on long range interacting neutrinos, LRI $v$, [solid yellow], and Planck $2018+$ BAO constraints [dotted purple]. We show the marginalized $2 \sigma$ credible regions and 1-D posterior probability distributions for $\sum m_{v}$ and $\frac{g}{M_{\phi}}$.

range interactions with strength $g / M_{\phi} \sim 10^{3}-10^{5} \mathrm{eV}^{-1}$. As we see in Fig. 3, these parameter values can be explored by EUCLID, allowing to test this hypothesis.

\section{Conclusions}

In this work, we have consistently explored the cosmology of neutrinos endowed with a scalarmediated long range interaction. We have also performed an analysis of current and near future cosmological data.

We have shown that long-range interacting neutrinos can behave as relativistic even for temperatures well below their mass, and have an equation of state that dramatically differs from the standard assumptions. As a consequence, there is no cosmological mass bound with present data, and a very interesting complementarity between the KATRIN experiment, neutrino oscillation experiments, and the future EUCLID survey emerges. To facilitate future studies of this complementarity, or applications of our formalism to other fermions, we have made our code publicly available [11].

Altogether, there is a bright future for laboratory and cosmological neutrino experiments that will shed light on the neutrino mass scale and the possible presence of long-range interactions.

\section{References}

[1] P. S. Pasquini and O. L. G. Peres, Bounds on Neutrino-Scalar Yukawa Coupling, Phys. Rev. D 93 (2016) 053007 [1511.01811]. [Erratum: Phys.Rev.D 93, 079902 (2016)].

[2] J. F. Beacom, N. F. Bell and S. Dodelson, Neutrinoless universe, Phys. Rev. Lett. 93 (2004) 121302 [astro-ph/0404585]. 
[3] Z. Chacko, L. J. Hall, T. Okui and S. J. Oliver, CMB signals of neutrino mass generation, Phys. Rev. D 70 (2004) 085008 [hep-ph/0312267].

[4] S. Hannestad, Structure formation with strongly interacting neutrinos - Implications for the cosmological neutrino mass bound, JCAP 02 (2005) 011 [astro-ph/0411475].

[5] N. F. Bell, E. Pierpaoli and K. Sigurdson, Cosmological signatures of interacting neutrinos, Phys. Rev. D 73 (2006) 063523 [astro-ph/0511410].

[6] E. Di Valentino, C. Bøehm, E. Hivon and F. R. Bouchet, Reducing the $H_{0}$ and $\sigma_{8}$ tensions with Dark Matter-neutrino interactions, Phys. Rev. D 97 (2018) 043513 [1710. 02559].

[7] C. D. Kreisch, F.-Y. Cyr-Racine and O. Doré, Neutrino puzzle: Anomalies, interactions, and cosmological tensions, Phys. Rev. D 101 (2020) 123505 [1902 .00534].

[8] M. Escudero and S. J. Witte, A CMB search for the neutrino mass mechanism and its relation to the Hubble tension, Eur. Phys. J. C 80 (2020) 294 [1909. 04044].

[9] B. Dasgupta and J. Kopp, Cosmologically Safe eV-Scale Sterile Neutrinos and Improved Dark Matter Structure, Phys. Rev. Lett. 112 (2014) 031803 [1310.6337].

[10] S. Hannestad, R. S. Hansen and T. Tram, How Self-Interactions can Reconcile Sterile Neutrinos with Cosmology, Phys. Rev. Lett. 112 (2014) 031802 [1310 . 5926].

[11] I. Esteban and J. Salvado, Long Range Interactions in Cosmology: Implications for Neutrinos, JCAP 05 (2021) 036 [2101.05804].

[12] Planck collaboration, Planck 2018 results. VI. Cosmological parameters, Astron. Astrophys. 641 (2020) A6 [1807.06209].

[13] F. Beutler, C. Blake, M. Colless, D. Jones, L. Staveley-Smith, L. Campbell et al., The $6 d F$ Galaxy Survey: Baryon Acoustic Oscillations and the Local Hubble Constant, Mon. Not. Roy. Astron. Soc. 416 (2011) 3017 [1106. 3366].

[14] A. J. Ross, L. Samushia, C. Howlett, W. J. Percival, A. Burden and M. Manera, The clustering of the SDSS DR7 main Galaxy sample - I. A 4 per cent distance measure at $z=0.15$, Mon. Not. Roy. Astron. Soc. 449 (2015) 835 [1409. 3242].

[15] BOSS collaboration, The clustering of galaxies in the completed SDSS-III Baryon Oscillation Spectroscopic Survey: cosmological analysis of the DR12 galaxy sample, Mon. Not. Roy. Astron. Soc. 470 (2017) 2617 [1607.03155].

[16] I. Esteban, M. Gonzalez-Garcia, M. Maltoni, T. Schwetz and A. Zhou, The fate of hints: updated global analysis of three-flavor neutrino oscillations, JHEP 09 (2020) 178 [2007.14792]. NuFIT 5.0 (2020), www. nu-fit.org.

[17] KATRIN collaboration, Improved Upper Limit on the Neutrino Mass from a Direct Kinematic Method by KATRIN, Phys. Rev. Lett. 123 (2019) 221802 [1909. 06048].

[18] EUCLID collaboration, Euclid Definition Study Report, 1110. 3193. 